\title{
Item Desirability Matching in Forced-choice Test Construction
}

\author{
Goran Pavlov ${ }^{1,2}$, Dexin Shi ${ }^{1}$, Alberto Maydeu-Olivares ${ }^{1,2}$, and Amanda Fairchild ${ }^{1}$ \\ ${ }^{1}$ University of South Carolina \\ ${ }^{2}$ University of Barcelona
}

Correspondence concerning this article should be addressed to Goran Pavlov, Department of Psychology, University of South Carolina, Barnwell College, 1512 Pendleton Street, Columbia, SC, 29208. Email: pavlovg@mailbox.sc.edu

This is the final author version (before journal's typesetting and copyediting) of the following article: Pavlov, G., Shi, D., Maydeu-Olivares, A., \& Fairchild, A. (2021). Item Desirability Matching in Forced-choice Test Construction. Personality and Individual Differences.

This paper is not the copy of record and may not exactly replicate the final, authoritative version of the article. Please do not copy or cite without authors' permission. The final article will be available, upon publication, via its DOI: 10.1016/j.paid.2021.111114. 


\begin{abstract}
The forced-choice method has been proposed as a viable strategy to prevent socially desirable responding (SDR) on self-report non-cognitive measures. The ability of the method to eliminate SDR stems from matching items that are perceived as equally desirable into forced-choice itemblocks. The gold standard in quantifying similarity between items in terms of desirability has been the "mean difference index", that is, the absolute difference between items' mean desirability ratings. This index relies on the assumption that items have one true desirability value, as efficiently and unbiasedly estimated by their respective means, and may fail if this assumption does not hold. To circumvent this issue, we propose indexing similarity between items in terms of desirability with several robust measures of absolute agreement (i.e., inter-item agreement indices). Using an empirical example, we show that relying on the mean difference index may lead to suboptimal forced-choice item-block assembly by matching items with a relatively poor inter-item agreement with respect to desirability. $\mathrm{R}$ code for computing the proposed agreement indices on a set of desirability ratings is provided, as are recommendations for applied researchers.
\end{abstract}

Keywords: forced-choice, desirability matching, socially desirable responding, faking, inter-item agreement 


\section{Introduction}

Self-report is a commonly used method for measuring non-cognitive constructs (e.g., personality, attitudes, interests) across a variety of research and applied settings. The popularity of self-report stems not only from its potential benefits over alternatives, such as practicality and cost, but also from an implicit expectation that respondents will tend to respond diligently when answering questions about themselves (Paulhus \& Vazire, 2007). By far, the most commonly used self-report measure format is the single-stimulus format, more commonly known as the Likert-type or rating format. When the rating format is used, respondents are typically asked to describe themselves or to indicate their agreement with a set of indicator items (e.g., statements or adjectives) on a rating scale with predetermined response options.

Notwithstanding advantages, measures in the rating format are susceptible to several systematic response biases, broadly defined as "non-test-relevant response determinants" (Crowne \& Marlowe, 1960, p. 394), which may represent a serious threat to assessment validity. Response biases are commonly subsumed into two broad groups: response styles and socially desirable responding (SDR; Wetzel et al., 2016). Response styles represent response biases unrelated to the item content, such as acquiescence (the tendency to choose response options stating agreement), extreme responding (the tendency to choose extreme response options), or midpoint responding (the tendency to choose midpoint or neutral response options). In contrast, SDR refers to the tendency to provide responses that result in a favorable picture of oneself, and thus directly relates to item content (Paulhus, 1984). SDR can be either unintentional, in which case it is referred to as self-deception or self-enhancement, or deliberate, commonly encountered 
in high-stakes assessment settings (e.g., personnel selection) and referred to as impression management or faking.

One of the methods for countering response biases has been to utilize alternative item formats such as the forced-choice response format. The forced-choice format consists of a series of item-blocks, typically composed of two to five items measuring the same (i.e., unidimensional forced-choice) or different (i.e., multidimensional forced-choice) constructs. Depending on the specific forced-choice design, respondents are either asked to 1) choose an item within each block that is more descriptive of them, 2) choose the most descriptive and the least descriptive item, or 3) provide a full ranking of items within each forced-choice block. By design, forcedchoice addresses several response styles and research to date has demonstrated that it may eliminate midpoint/extreme responding and acquiescence (see e.g., Cheung \& Chan, 2002).

The forced-choice response format has been also thought of as potentially advantageous for preventing SDR, given that it does not allow respondents to express (dis)agreement with all (un)desirable items within an item-block. Nevertheless, if the items composing the forced-choice blocks are perceived to be of different desirability, the format itself may not be sufficient to prevent SDR and may even enhance it. Therefore, as suggested by Edwards (1953), items comprising forced-choice item-blocks should be balanced or matched in terms of their perceived desirability. The forced-choice format coupled with desirability matching of forced-choice blocks has been commonly referred to as the forced-choice method (e.g., Feldman \& Corah, 1960; Rothstein \& Goffin, 2006). It has been presumed that measures developed using this method may prevent SDR by forcing respondents to answer more accurately and in a selfdescriptive manner (Dilchert \& Ones, 2011; Rothstein \& Goffin, 2006; Saltz et al., 1962). 
To date, the forced-choice as a SDR prevention method has received considerable research attention, especially from researchers within the area of personnel selection. Specifically, of focal interest to researchers has been to ascertain if and to what extent measures developed with the forced-choice method can minimize or reduce an overall inflation of test scores (e.g., Bass, 1957; Stark et al., 2014; Wetzel et al., 2021), keep an adequate ordering of test respondents (e.g., Bartlett \& Doorley, 1967; Heggestad et al., 2006; Longstaff \& Jurgensen, 1953), and/or better preserve criterion-related validity than their rating format counterparts (e.g., Christiansen et al., 2005; Jackson et al., 2000; Vasilopoulos et al., 2006). Notwithstanding the sound rationale behind the forced-choice method, these research efforts have produced conflicting and overall limited evidence in support of the methods' property to address SDR.

One plausible explanation for the lack of stronger evidence in support of the method preventing SDR is that forced-choice measures used in previous research have not been optimally matched in terms of desirability, thus allowing respondents to make desirability driven choices. Despite this potential concern, efforts to explicitly and systematically investigate and/or advance methods for desirability matching have been scarce (e.g., Converse et al., 2010). In this article, we seek to address this outstanding need by discussing existing methods and proposing a novel desirability matching approach. The current work seems particularly timely given recent breakthroughs in modeling and scoring forced-choice responses (Brown \& Maydeu-Olivares, 2011; Stark et al., 2005) and an emerging popularity of forced-choice measures in organizational, educational, clinical, and other substantive domains (e.g., Anguiano-Carrasco et al., 2015; Dueber et al., 2019; Guenole et al., 2018; Murano et al., 2021; Ng et al., 2021; Stark et al., 2014; Walton et al., 2020; Watrin et al., 2019; Wetzel \& Frick, 2020). 


\section{The Forced-choice Method and SDR}

Consider the following three personality items from the International Personality Item Pool (IPIP: Goldberg, 1992), intended to measure, respectively, Conscientiousness, Neuroticism, and Openness to Experience dimension of the Big Five model (Goldberg, 1990):

Am always prepared

Get stressed out easily

\section{Catch on to things quickly}

When the items above are administered using the rating response format, respondents are commonly asked to express their agreement with each of the items separately, one at the time, on a rating scale with a predetermined number of response categories. This rating format is highly susceptible to SDR because respondents can easily move their responses in a desirable direction. Respondents may self-enhance by unconscientiously expressing higher agreement than they really have with items that appear more favorable (i.e., the top and bottom items), and higher disagreement with items that appear less favorable (i.e., the middle item. The SDR bias may be exacerbated if the assessment is performed in high-stakes settings, for instance, as part of the employee selection process. Specifically, some job applicants might be motivated to alter their responses (i.e., fake) in a way that maximizes their chances of advancing to the next stage of the selection process and getting a job offer, by offering responses that align with a desirable image for the target job.

Alternatively, the forced-choice response format could be employed to administer the three items above. In such case, all three items would be presented simultaneously within the same forced-choice item-block, and respondents could be asked to choose one item that describes them the most ("most like me") and one that describes them the least ("least like me"). 
This format may counter SDR because respondents no longer have an option to agree with all desirable items and disagree with all undesirable ones. In the example above, a respondent may select only one of the two desirable items, that is, the top and the bottom item, as "most like me". Nevertheless, the forced-choice format alone need not suffice to prevent desirability driven responding entirely. A respondent can still self-enhance/fake by selecting the middle item as "least like me" regardless of their actual standing on the underlying trait. Thus, to be able prevent SDR, all items within the same item-block should elicit sufficiently similar desirability evaluations.

\section{Desirability Matching Procedures}

To construct a forced-choice measure resistant to SDR, it is essential to identify itemcombinations for which differences in desirability evaluations between items are sufficiently similar across test takers. Typically, item desirability matching relies on empirically retrieved item desirability ratings (Chernyshenko et al., 2009). Such ratings may be obtained with several approaches, which may vary with respect to the composition of the desirability sample and also the instruction set used to obtain them. With respect to the former, the desirability sample may derive from the population in which the assessment is targeted (e.g., Usami et al., 2016; Vasilopoulos et al., 2006), may make use of domain experts specific to the assessment content (e.g., Chernyshenko et al., 2009; Jackson et al., 2000), or both (e.g., Drasgow et al., 2012). With respect to the latter, a desirability study may be designed to provide respondents with a neutral (i.e., straight-take) instruction set (e.g., Guenole et al., 2018; Watrin et al., 2019), a set explicitly manipulated to incentivize SDR (e.g., Drasgow et al., 2012; Naemi et al., 2014; Stark et al., 2005), or to ask respondents to explicitly rate desirability of each item under consideration (e.g., Chernyshenko et al., 2009; Christiansen et al., 2005; Vasilopoulos et al., 2006; Wetzel et al., 
2021). Desirability ratings may be obtained with respect to general social desirability (e.g., Usami et al., 2016; Wetzel et al., 2021) or be context specific (e.g., Converse et al., 2010; Drasgow et al., 2012; Jackson et al., 2000). Research to date suggests that different approaches may produce non-negligible differences in desirability ratings (e.g., Drasgow et al., 2012; Hedberg \& Baxter, 1963) and influence the effectiveness of desirability matching (e.g., Converse et al., 2010), though there are no empirically driven guidelines in the literature as to which approach should be championed regardless of context.

When desirability ratings are collected, items are matched according to the similarity of their desirability estimates. Most commonly, desirability ratings are averaged across respondents to arrive at one desirability estimate for each item. Then, absolute differences in mean desirability ratings are computed between each item-pair, with smaller values indicating closer matches. These estimates are then used to select item-pairs for inclusion within a forced-choice item-block, commonly in conjunction with other psychometric criteria, such as, item discrimination, location, and content. Most often, test developers proceed by specifying an $a$ priori absolute mean difference value as the maximum acceptable level of dissimilarity between items. Item-pairs with absolute mean difference estimates smaller than the cutoff are considered to consist of items of "sufficiently" similar desirability. Though different cutoff values have been used in the literature, values of 0.5 units (Edwards, 1957; Vasilopoulos et al., 2006) and 1 unit (e.g., Chernyshenko et al., 2009; Drasgow et al., 2012) are most common, with the magnitude of these cutoffs interpreted relative to the number of categories of the rating scale used.

\section{The Mean Difference Index}

The mean difference index represents the key metric and the gold standard in item desirability matching. Formally put, the mean difference index is given by 


$$
D=\left|M_{a}-M_{b}\right|
$$

where $M_{a}$ and $M_{b}$ are mean desirability ratings for items $a$ and $b$, respectively. The mean difference index, $D$, quantifies similarity between two items in terms of desirability in units of the rating scale used (Kilmann \& Thomas, 1977). Item-pairs with smaller mean difference values are considered to be closer in terms of perceived desirability and accordingly better matches, with $D=0$ indicating an exact match (Feldman \& Corah, 1960).

We briefly demonstrate the application of $D$ with a hypothetical example in Table 1 . Consider that 10 respondents are asked to evaluate the desirability of 5 items on a 7-point rating scale ranging from strongly undesirable (coded with "1") to strongly desirable (coded with "7"). Item desirability means and associated standard deviations for each item are presented. Using Equation (1), $D$ can be easily computed between each of the 10 possible item-pairs. Reviewing the column means in the table, we see that $\hat{D}$ for each of the 10 item-pairs is exactly 0 . Accordingly, each pair may be considered an exact match in terms of desirability and equally appropriate for forced-choice block assembly.

In essence, the mean difference index averages desirability ratings across respondents within items, and then quantifies absolute differences in mean ratings between items. To the extent that there is no systematic error variance about the mean desirability ratings, this method is both simple and effective. We can consider, however, scenarios that may result in substantive intrasample heterogeneity of opinion regarding desirability of items and lead to nonrandom error variance about the mean desirability ratings. For example, some of the heterogeneity may be due to gender differences in perceived congruence of item content with respect to societal (or organizational) norms and expectations (see e.g., Feingold, 1994). To the extent that there is a substantial difference in perceived desirability of the items for individual respondents more 
generally (i.e., systematic or not), the resulting mean difference index may be statistically inefficient (Nederhof, 1985).

\begin{tabular}{cccccc}
\hline & \multicolumn{5}{c}{ Item } \\
\cline { 2 - 6 } Respondent & i1 & i2 & i3 & i4 & i5 \\
\hline r1 & 6 & 5 & 4 & 6 & 7 \\
r2 & 5 & 5 & 6 & 4 & 4 \\
r3 & 5 & 5 & 4 & 6 & 6 \\
r4 & 5 & 5 & 6 & 4 & 4 \\
r5 & 5 & 5 & 3 & 7 & 7 \\
r6 & 5 & 5 & 7 & 3 & 3 \\
r7 & 5 & 5 & 3 & 7 & 7 \\
r8 & 5 & 5 & 7 & 3 & 3 \\
r9 & 5 & 5 & 4 & 7 & 7 \\
r10 & 5 & 6 & 7 & 4 & 3 \\
\hline M & 5.10 & 5.10 & 5.10 & 5.10 & 5.10 \\
SD & 0.32 & 0.32 & 1.66 & 1.66 & 1.85 \\
\hline
\end{tabular}

Table 1

Example Desirability Ratings on a 7-point Rating Scale and Summary Statistics by Item

Note. $\mathrm{M}=$ mean rating; $\mathrm{SD}=$ standard deviation.

Concerns regarding respondents' heterogeneity in their rating of items' desirability was recognized in early applications of the forced-choice method (see e.g., Maher, 1959; Norman, 1963). Norman (1963) argued that one way to circumvent this issue and improve desirability matching would be to consider only items with near zero desirability variances; this recommendation has been largely neglected in consequent attempts to construct SDR-resistant forced-choice measures, however. An exception was the work by Vasilopoulos et al. (2006), who devised a stepwise approach to item matching that first removed items with variability in desirability ratings above 1 standard deviation (SD) before computing $D$ between all remaining items. Similar strategies to incorporate an assessment of respondent consensus in quantifying 
item desirability could be invoked with a variety of interrater agreement (IRA) indices appropriate for a single item, such as $r_{w g}, a_{w g}$, and the AD index (James et al., 1984; Brown \& Hauenstein, 2005; and Burke et al., 1999, respectively).

Returning to the example in Table 1, it can be observed that the variability of desirability ratings is relatively low for items i1 and i2, and relatively high for items i3 through i5. In other words, there appears to be a strong consensus among respondents when it comes to desirability of the former but not the latter group of items. It can also be observed that the $\hat{D}$ value between items with strong consensus, i.e., i1 and i2, appropriately quantifies similarity in desirability ratings across respondents but does not do so uniformly for items that lack consensus (i.e., i3 through i5). Although $D$ flags items i3 and i4 as perfectly matched in terms of desirability ( $\hat{D}=$ 0), items comprising the pair have received a markedly different desirability rating by every respondent in the desirability sample. On the other hand, $D$ appropriately quantifies similarity between items i4 and i5, despite the considerable disagreement regarding desirability of both (SDs > 1.5). Overall, though considering only items for which a "sufficient" consensus regarding desirability is observed may ensure that $D$ will return a relatively appropriate similarity value, this approach has been shown to be overly conservative by removing an excessive number items from consideration (Vasilopoulos et al., 2006).

\section{Inter-item Agreement (IIA) Indices}

As discussed previously, the goal in item desirability matching is to identify combinations of items that will be perceived as equally desirable by test respondents. If matching relies on desirability ratings, this goal may be attained by identifying items that have received the same or, at least, very similar desirability rating by each respondent in the desirability sample. Therefore, of main interest is the magnitude of within-respondent agreement (i.e., intra-rater 
agreement) with respect to desirability evaluations of each item-combination under consideration. We refer to intra-rater agreement indices here as inter-item agreement (IIA) indices, as they reflect the extent to which items comprising an item-combination are interchangeable, i.e., indistinguishable in terms of desirability. In the following, we discuss only IIA indices for the case of two items to provide meaningful comparison with the $D$ index.

Historically, the main choices for indexing agreement have been intraclass correlation

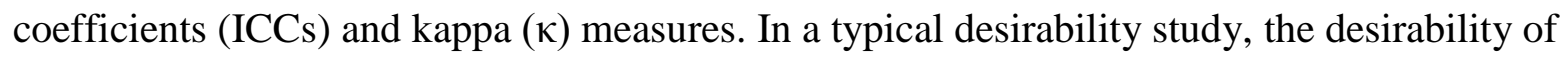
each of I items is evaluated by the same sample of $\mathrm{R}$ respondents. Given two items, $a$ and $b$, the IIA can be estimated as a two-way ICC given by

$$
I C C_{[2,1]}=\frac{M S_{R}-M S_{E}}{M S_{R}+M S_{E}+2 \frac{M S_{I}-M S_{E}}{R}},
$$

where $M S_{R}$ is the mean square for rows (i.e., raters), $M S_{I}$ is the mean square for columns (i.e., items), and $M S_{E}$ is the mean square for error, all obtained from a two-way ANOVA (Shrout \& Fleiss, 1979). An ICC of 1 indicates perfect agreement, 0 indicates absence of agreement, and negative ICC values indicate a "systematic" disagreement.

IIA can be alternatively indexed using kappa $(\kappa)$ coefficients. Kappa is commonly defined as the proportion of observed agreement over and above what would be expected by chance. For ordinal/interval data and two raters (i.e., two items in this context), weighted kappa $\left(\kappa_{\mathrm{w}}\right)$ allows for partial agreement, such that disagreement on two adjacent rating categories is empirically quantified as less serious than disagreement on two distant categories (Cohen,1968). A rating difference of 4 (e.g., 2 vs. 6) between items, for example, is treated as more severe than 
a rating difference of 1 (e.g., 4 vs. 5), which would be considered as near agreement. Letting $q$ be the items' number of response alternatives, $\kappa_{\mathrm{w}}$ is given by

$$
\kappa_{w}=\frac{p_{o}-p_{e}}{1-p_{e}} \text {, with } p_{o}=\sum_{i=1}^{q} \sum_{j=1}^{q} p_{i j} w_{i j} \text { and } p_{e}=\sum_{i=1}^{q} \sum_{j=1}^{q} p_{i+1} p_{+j} w_{i j} \text {, }
$$

where $p_{o}$ is observed agreement, $p_{e}$ is agreement expected by chance, $p_{i j}$ is relative frequency in a two-way contingency table, $w_{i j}$ are weights that describe closeness of agreement between categories, and $p_{i+}$ and $p_{+j}$ are marginal probabilities for items $a$ and $b$ respectively. Several weighting schemes have been proposed in the literature, though the most widely used are linear, i.e., $w_{i j}=1-|i-j|(q-1)^{-1}$, and quadratic, i.e., $w_{i j}=1-(i-j)^{2}(q-1)^{-2}$ (see Gwet, 2014, for a review of different weighting schemes).

Kappa indices have corresponding forms of ICCs. For instance, a quadratically weighted $\kappa_{\mathrm{w}}$ is asymptotically equivalent to $\operatorname{ICC}_{[2,1]}$ (Fleiss \& Cohen, 1973; Schuster, 2004). Accordingly, $\kappa_{\mathrm{W}}$ is interpreted in a same way as ICC: 1 indicates perfect agreement, 0 indicates absence of agreement (i.e., equal to that expected by chance), and negative $\kappa_{\mathrm{w}}$ values indicate a systematic disagreement. Evaluation of the magnitude or strength of agreement has traditionally relied on some benchmarking scale (e.g., Fleiss, 1981; Landis \& Koch, 1977; Shrout, 1998). For instance, the most widely used benchmarking scale, proposed by Landis and Koch (1977), provides the following ranges: $<0$ poor, $[0 ; 0.20]$ slight, $[0.21 ; 0.40]$ fair, $[0.41,0.60]$ moderate, $[0.61,0.80]$ substantial, and $[0.81 ; 1.0]$ excellent or near perfect agreement. In applications, values above 0.70 are commonly considered to indicate adequate agreement (LeBreton \& Senter, 2008).

Notwithstanding their widespread use, it has been well documented that kappa and ICC indices may exhibit peculiar behavior and return misleading agreement values when the observed ratings indicate otherwise, e.g., strong agreement (e.g., Feinstein \& Cicchetti, 1990; Graham \& 
Jackson, 1993; Gwet, 2008; LeBreton et al., 2003; Quarfoot \& Levine, 2016; Warrens, 2012).

Several robust agreement indices have been proposed in the literature to overcome drawbacks of ICCs and kappas. One such measure, the weighted Brennan-Prediger index (e.g., Brennan \& Prediger, 1981; Gwet, 2014) is given by

$$
B P_{w}=\frac{p_{o}-p_{e}}{1-p_{e}}, \text { with } p_{e}=\frac{T_{w}}{q^{2}} \text { and } T_{w}=\sum_{i=1}^{q} \sum_{j=1}^{q} w_{i j},
$$

where $T_{w}$ is the sum over all weight values $w_{i j}$, and $p_{o}$ is given in (3). While $B P_{w}$ has the typical kappa-type form, $p_{e}$ is estimated differently: The Brennan-Prediger index adopts a uniform distribution for the probability of agreement by chance and is thus independent of the observed marginal probabilities. Gwet $(2008,2014)$ introduced agreement coefficients, AC, as alternatives to unweighted and weighted $\kappa$. The weighted version, $\mathrm{AC}_{2}$, is recommended for analyzing ordinal, interval, and ratio data (Gwet, 2014) and is given by

$$
A C_{2}=\frac{p_{o}-p_{e}}{1-p_{e}}, \text { with } p_{e}=\frac{T_{w}}{q(q-1)} \sum_{k=1}^{q} p_{k}\left(1-p_{k}\right) \text { and } p_{k}=\frac{p_{k+}+p_{+k}}{2}
$$

where $p_{k}$ is the average of the corresponding marginal probabilities and $p_{o}$ is given in (3). AC is a generalization of the BP index that averages marginal probabilities across response categories, with a goal of more adequately estimating the overall agreement by chance (i.e., $p_{e}$; Gwet, 2008). The robustness of both $\mathrm{AC}$ and $\mathrm{BP}$ indices, as compared to $\kappa$ or ICC estimates, has received notable support in the literature to date (e.g., Quarfoot \& Levine, 2016; Tran et al., 2020; Wongpakaran et al., 2013), and these indices can be conveniently calculated in widely available statistical software packages, such as R or Stata (Klein, 2018).

We illustrate and compare the IIA estimates indices discussed above by applying them to the example data originally presented in Table 1 . Specifically, Table 2 contains estimates for the: 
a) mean difference index $(D)$; b) intraclass correlation coefficient $\left(\operatorname{ICC}_{[2,1]}\right)$ linearly weighted kappa $\left(\kappa_{l}\right), \mathrm{BP}\left(B P_{l}\right)$, and $\mathrm{AC}_{2}\left(\mathrm{AC}_{l}\right)$ indices; and d) quadratically weighted kappa $\left(\kappa_{q}\right), \mathrm{BP}\left(B P_{q}\right)$, and $\mathrm{AC}_{2}\left(\mathrm{AC}_{q}\right)$ indices across all possible item-pairs. It can be observed that the $\mathrm{AC}$ and $\mathrm{BP}$ indices identified item-pairs $\{\mathrm{i} 1, \mathrm{i} 2\}$ and $\{\mathrm{i} 4, \mathrm{i} 5\}$ as most similar in terms of desirability. An inspection of the raw ratings in these pairs in Table 1 indicates each item received the same, or very similar, desirability rating by each of 10 respondents in the sample. Furthermore, the BP and $\mathrm{AC}$ indices identified item-pairs $\{\mathrm{i} 3, \mathrm{i} 4\}$ and $\{\mathrm{i} 3, \mathrm{i} 5\}$ as pairs with the lowest agreement. This disagreement can be observed in the raw ratings of these pairs in Table 1. For instance, even though items i3 and i4 have an identical mean desirability estimate (M; Table 1), they received a markedly different desirability rating by each respondent in the desirability sample.

\begin{tabular}{crrrrrrrr}
\hline Item-pair & $\hat{D}$ & \multicolumn{1}{c}{$I \hat{C} C_{[2,1]}$} & \multicolumn{1}{c}{$\hat{\kappa}_{l}$} & \multicolumn{1}{c}{$\hat{\kappa}_{q}$} & \multicolumn{1}{c}{$B \hat{P}_{l}$} & \multicolumn{1}{c}{$B \hat{P}_{q}$} & \multicolumn{1}{l}{$A \hat{C}_{l}$} & $A \hat{C}_{q}$ \\
\hline$\{\mathrm{i} 1, \mathrm{i} 2\}$ & 0 & -0.125 & -0.111 & -0.111 & 0.913 & 0.975 & 0.962 & 0.993 \\
$\{\mathrm{i} 1, \mathrm{i} 3\}$ & 0 & -0.096 & -0.067 & -0.085 & 0.300 & 0.650 & 0.444 & 0.776 \\
$\{\mathrm{i} 1, \mathrm{i} 4\}$ & 0 & 0.077 & 0.067 & 0.070 & 0.388 & 0.700 & 0.514 & 0.808 \\
$\{\mathrm{i} 1, \mathrm{i} 5\}$ & 0 & 0.131 & 0.059 & 0.119 & 0.300 & 0.650 & 0.449 & 0.779 \\
$\{\mathrm{i} 2, \mathrm{i} 3\}$ & 0 & 0.161 & 0.067 & 0.147 & 0.388 & 0.725 & 0.514 & 0.824 \\
$\{\mathrm{i} 2, \mathrm{i} 4\}$ & 0 & -0.096 & -0.067 & -0.085 & 0.300 & 0.650 & 0.444 & 0.776 \\
$\{\mathrm{i} 2, \mathrm{i} 5\}$ & 0 & -0.149 & -0.059 & -0.132 & 0.213 & 0.550 & 0.380 & 0.715 \\
$\{\mathrm{i} 3, \mathrm{i} 4\}$ & 0 & -1.205 & -0.724 & -0.968 & -0.313 & -0.225 & -0.074 & 0.171 \\
$\{\mathrm{i} 3, \mathrm{i} 5\}$ & 0 & -1.210 & -0.739 & -0.971 & -0.400 & -0.375 & -0.128 & 0.095 \\
$\{\mathrm{i} 4, \mathrm{i} 5\}$ & 0 & 0.968 & 0.891 & 0.964 & 0.913 & 0.975 & 0.929 & 0.984 \\
\hline
\end{tabular}

Table 2

Estimates of D and IIA Indices for All Possible Item-pairs in Example in Table 1

Note. $\mathrm{N}=10 ; D=$ mean difference index; $\mathrm{ICC}_{[2,1]}=$ intraclass correlation coefficient (Shrout \& Fleiss, 1979); $\kappa_{l}=$ linearly weighted kappa (Cohen, 1968); $\kappa_{q}=$ quadratically weighted kappa (Cohen, 1968); $B P_{l}=$ linearly weighted BP index (Brennan \& Prediger, 1981; Gwet, 2014); $B P_{q}$ $=$ quadratically weighted BP index (Brennan \& Prediger, 1981; Gwet, 2014); $A C_{l}=$ linearly weighted $\mathrm{AC}_{2}$ index (Gwet, 2008; 2014); $A C_{q}$ = quadratically weighted $\mathrm{AC}_{2}$ index (Gwet, 2008; 2014).For $\mathrm{D}$, values of $\leq 0.5$ are highlighted (Vasilopoulos et al., 2006); for $\operatorname{ICC}_{[2,1]}$ through $A C_{q}$, values of $\geq 0.7$ are highlighted (LeBreton \& Senter, 2008). 
It can also be observed in Table 2 that $\mathrm{ICC}_{[2,1]}$ and $\kappa_{\mathrm{w}}$ indices may yield unreasonably low estimates of otherwise high IIA. This tendency was most severe for item-pair $\{\mathrm{i} 1, \mathrm{i} 2\}$, for which $\mathrm{ICC}_{[2,1]}$ and $\kappa_{\mathrm{w}}$ returned a negative value. Finally, the tables reveal that the $D$ index provided appropriate similarity estimates only for item-pairs $\{\mathrm{i} 1, \mathrm{i} 2\}$ and $\{\mathrm{i} 4, \mathrm{i} 5\}$, while failing most severely for item-pairs $\{i 3, i 4\}$ and $\{i 3, i 5\}$. In short, these results suggest that 1) $D$ may fail to appropriately quantify similarity of desirability ratings between items, and 2) among IIA options, $\mathrm{AC}$ or BP measures seem to adequately quantify similarity between items and overcome issues inherent in ICC and $\kappa$ measures.

\section{An Empirical Example}

In the example data provided in Table 1, we used a convenient constellation of desirability ratings to illustrate our arguments more effectively and to depict circumstances under which the $D$ index may fail. However, it is possible that the chosen scenario was contrived and that such extreme circumstances will not be encountered in practical applications. Accordingly, we supplement it with analysis performed on desirability ratings obtained with a typical desirability study to examine 1) the correspondence between the $D$ and IIA indices, and 2) the extent to which item selection and item matching may be affected by the choice of item similarity metric in real data. In the interest of simplicity, we focus only on the BP and AC indices as IIA comparators in the example given support for their robustness in the literature.

\subsection{Sample}

The study participants were undergraduate students enrolled in various programs at a large public university in the Eastern part of the Republic of China. Six hundred and fourteen participants completed the study. We made use of two strategies to monitor random responding 
(Meade \& Craig, 2012). First, we excluded participants who completed the survey in 5 minutes or less given this was deemed an unreasonable response time given the questionnaire. Second, we conducted manipulation check to control for random responding. The manipulation check consisted of a single rating of the extent respondents had followed the instructions while responding to questionnaire. These strategies resulted in the removal of 29 participants who responded negatively to the manipulation check and 69 participants who completed the study too fast. The number of participants retained for the analyses was 516 . The average participant age was 21.2 years. Three hundred and ninety-seven participants $(76.9 \%)$ were female.

\subsection{Measures}

We used 120 items from the Chinese version of the IPIP-NEO-120 scale (Johnson, 2014). The scale measures Big Five personality dimensions, with 24 items per dimension. A 7-point rating scale was used with the following anchors: completely disagree, disagree, slightly disagree, neither disagree nor agree, slightly agree, agree, and completely agree. The responses were coded from $1=$ completely disagree to $7=$ completely agree . Extreme response anchors were used to ensure that the response scale covers respondents' entire response space.

\subsection{Procedure}

The study was administered online. Desirability data collection relied on a fake-good study (Drasgow et al., 2012) and included a specific instruction to fake (Converse et al., 2010). After providing informed consent, respondents were asked to imagine that they were applying for a high-school math teacher job opening. The target job was chosen under the assumption that university students should be well acquainted with its requirements. In addition, for this target job, all Big Five dimensions seemed relevant and desirable (for Neuroticism, the opposite was true). Respondents were instructed to respond to the questionnaire in a way that would convince 
the employer that they were an ideal candidate for the specified job position. Items were presented to respondents in a random order. After responding to all IPIP-NEO-120 items, respondents were asked to respond to the manipulation check.

\subsection{Results}

In Table 3, we present summary statistics for all 120 IPIP items. Mean desirability values ranged from 2.138 to 6.380 . As expected, positively keyed items had higher mean desirability values, and the opposite was true for Neuroticism.

To evaluate similarity of desirability ratings between all possible $n=7,140$ item-pairs, we computed the mean difference index $(D)$ and 4 IIA statistics: the linearly and quadratically weighted BP indices $\left(B P_{l}\right.$ and $\left.B P_{q}\right)$, and the linearly and quadratically weighted AC indices $\left(A C_{l}\right.$ and $A C_{q}$ ). All computations were performed using the irrCAC and combinat packages in $\mathrm{R}(\mathrm{R}$ Core Team, 2018). For readers interested in computing these indices in their own research, we provide $\mathrm{R}$ code in materials supplementary to this article.

Descriptive statistics and intercorrelations between all five matching indices are presented in Table 4. We observe in this table that the item-pairs varied dramatically with respect to similarity in terms of desirability. $\hat{D}$ values ranged from 0 , indicating an exact match, to 4.242, indicating a difference in desirability between items of over 4 units on the (7-point) rating scale. Ranges of estimated IIA values differed depending on the index used, but in all cases IIA ranged from near perfect agreement (>0.8) to systematic disagreement (negative values) between items. 


\begin{tabular}{|c|c|c|c|c|c|c|c|c|c|c|c|}
\hline I & $\mathrm{T}$ & $\mathrm{M}$ & $\mathrm{SD}$ & $\mathrm{I}$ & $\mathrm{T}$ & $\mathrm{M}$ & SD & $\mathrm{I}$ & $\mathrm{T}$ & $\mathrm{M}$ & SD \\
\hline 57 & $E$ & 6.380 & 0.798 & 72 & $E$ & 5.225 & 1.339 & 60 & $\mathrm{C}(-)$ & 3.295 & 1.787 \\
\hline 15 & C & 6.368 & 0.683 & 22 & E & 5.219 & 1.321 & 36 & $\mathrm{~N}$ & 3.285 & 1.845 \\
\hline 14 & A & 6.316 & 0.814 & 4 & A & 5.213 & 1.146 & 66 & $\mathrm{~N}$ & 3.269 & 1.822 \\
\hline 44 & A & 6.306 & 0.745 & 101 & $\mathrm{~N}(-)$ & 5.202 & 1.384 & 11 & $\mathrm{~N}$ & 3.242 & 1.815 \\
\hline 12 & $\mathrm{E}$ & 6.266 & 0.830 & 17 & $\mathrm{E}$ & 5.167 & 1.366 & 69 & $\mathrm{~A}(-)$ & 3.203 & 1.719 \\
\hline 20 & $\mathrm{C}$ & 6.260 & 0.795 & 42 & E & 5.095 & 1.401 & 16 & $\mathrm{~N}$ & 3.190 & 1.766 \\
\hline 8 & $\mathrm{O}$ & 6.227 & 0.830 & 96 & $\mathrm{~N}(-)$ & 5.083 & 1.661 & 30 & $\mathrm{C}(-)$ & 3.155 & 1.702 \\
\hline 87 & $\mathrm{E}$ & 6.176 & 0.982 & 37 & $\mathrm{E}$ & 5.066 & 1.620 & 80 & $C(-)$ & 3.136 & 1.657 \\
\hline 38 & $\mathrm{O}$ & 6.112 & 0.861 & 13 & $\mathrm{O}$ & 5.025 & 1.682 & 107 & $\mathrm{E}(-)$ & 3.132 & 1.787 \\
\hline 35 & $\mathrm{C}$ & 6.079 & 0.920 & 59 & A & 4.919 & 1.613 & 71 & $\mathrm{~N}$ & 3.110 & 1.749 \\
\hline 58 & $\mathrm{O}$ & 6.052 & 1.202 & 31 & $\mathrm{~N}$ & 4.845 & 1.795 & 73 & $\mathrm{O}(-)$ & 3.099 & 1.615 \\
\hline 45 & $\mathrm{C}$ & 6.050 & 0.958 & 48 & $\mathrm{O}(-)$ & 4.816 & 1.597 & 68 & $\mathrm{O}(-)$ & 3.068 & 1.688 \\
\hline 117 & $\mathrm{E}$ & 6.039 & 1.038 & 7 & $\mathrm{E}$ & 4.810 & 1.483 & 83 & $\mathrm{O}(-)$ & 3.060 & 1.756 \\
\hline 43 & $\mathrm{O}$ & 6.021 & 0.889 & 112 & $\mathrm{E}$ & 4.785 & 1.656 & 62 & $\mathrm{E}(-)$ & 3.041 & 1.733 \\
\hline 23 & $\mathrm{O}$ & 5.994 & 1.066 & 1 & $\mathrm{~N}$ & 4.731 & 1.769 & 102 & E(-) & 3.023 & 1.657 \\
\hline 10 & $\mathrm{C}$ & 5.988 & 1.118 & 32 & $\mathrm{E}$ & 4.641 & 1.742 & 94 & $\mathrm{~A}(-)$ & 2.998 & 1.535 \\
\hline 5 & $\mathrm{C}$ & 5.961 & 0.913 & 106 & $N(-)$ & 4.630 & 1.826 & 86 & $\mathrm{~N}$ & 2.936 & 1.698 \\
\hline 3 & $\mathrm{O}$ & 5.955 & 1.087 & 64 & A & 4.519 & 1.608 & 90 & $C(-)$ & 2.913 & 1.626 \\
\hline 63 & $\mathrm{O}$ & 5.921 & 1.149 & 84 & $\mathrm{~A}(-)$ & 4.428 & 1.498 & 70 & $C(-)$ & 2.897 & 1.783 \\
\hline 34 & A & 5.895 & 0.988 & 76 & $\mathrm{~N}$ & 4.421 & 1.924 & 21 & $\mathrm{~N}$ & 2.886 & 1.711 \\
\hline 29 & A & 5.855 & 1.072 & 24 & $\mathrm{~A}(-)$ & 4.409 & 1.538 & 82 & $\mathrm{E}$ & 2.864 & 1.662 \\
\hline 65 & $\mathrm{C}$ & 5.845 & 1.031 & 61 & $\mathrm{~N}$ & 4.203 & 1.876 & 78 & $\mathrm{O}(-)$ & 2.808 & 1.670 \\
\hline 27 & $\mathrm{E}$ & 5.829 & 1.200 & 67 & $\mathrm{E}(-)$ & 4.016 & 1.770 & 100 & $C(-)$ & 2.769 & 1.791 \\
\hline 55 & $\mathrm{C}$ & 5.808 & 1.169 & 115 & $C(-)$ & 3.802 & 1.760 & 108 & $\mathrm{O}(-)$ & 2.731 & 1.564 \\
\hline 33 & $\mathrm{O}$ & 5.806 & 1.256 & 46 & $\mathrm{~N}$ & 3.785 & 1.781 & 119 & $\mathrm{~A}(-)$ & 2.717 & 1.501 \\
\hline 18 & $\mathrm{O}$ & 5.789 & 1.177 & 49 & $\mathrm{~A}(-)$ & 3.779 & 1.970 & 41 & $\mathrm{~N}$ & 2.713 & 1.755 \\
\hline 118 & $\mathrm{O}(-)$ & 5.767 & 1.218 & 26 & $\mathrm{~N}$ & 3.692 & 1.863 & 104 & $\mathrm{~A}(-)$ & 2.700 & 1.602 \\
\hline 25 & $\mathrm{C}$ & 5.690 & 1.199 & 53 & $\mathrm{O}(-)$ & 3.638 & 1.841 & 109 & $\mathrm{~A}(-)$ & 2.628 & 1.644 \\
\hline 2 & $\mathrm{E}$ & 5.674 & 1.444 & 114 & $\mathrm{~A}(-)$ & 3.630 & 1.684 & 98 & $\mathrm{O}(-)$ & 2.601 & 1.560 \\
\hline 77 & $\mathrm{E}$ & 5.674 & 1.225 & 9 & $\mathrm{~A}(-)$ & 3.597 & 1.747 & 19 & $\mathrm{~A}(-)$ & 2.517 & 1.509 \\
\hline 28 & $\mathrm{O}$ & 5.649 & 1.436 & 91 & $\mathrm{~N}$ & 3.568 & 1.917 & 89 & $\mathrm{~A}(-)$ & 2.432 & 1.383 \\
\hline 95 & $\mathrm{C}$ & 5.527 & 1.291 & 92 & $\mathrm{E}(-)$ & 3.545 & 1.807 & 110 & $C(-)$ & 2.430 & 1.475 \\
\hline 111 & $\mathrm{~N}(-)$ & 5.403 & 1.326 & 56 & $\mathrm{~N}$ & 3.533 & 1.822 & 75 & $\mathrm{C}(-)$ & 2.411 & 1.467 \\
\hline 47 & $\mathrm{E}$ & 5.386 & 1.438 & 40 & $\mathrm{C}(-)$ & 3.500 & 1.955 & 105 & $C(-)$ & 2.407 & 1.468 \\
\hline 51 & $\mathrm{~N}(-)$ & 5.347 & 1.459 & 6 & $\mathrm{~N}$ & 3.496 & 1.749 & 99 & $\mathrm{~A}(-)$ & 2.405 & 1.394 \\
\hline 116 & $\mathrm{~N}(-)$ & 5.339 & 1.408 & 54 & $\mathrm{~A}(-)$ & 3.432 & 1.635 & 103 & $\mathrm{O}(-)$ & 2.403 & 1.428 \\
\hline 81 & $\mathrm{~N}(-)$ & 5.335 & 1.375 & 113 & $\mathrm{O}(-)$ & 3.399 & 1.876 & 120 & $C(-)$ & 2.277 & 1.425 \\
\hline 50 & $\mathrm{C}$ & 5.333 & 1.231 & 88 & $\mathrm{O}(-)$ & 3.386 & 1.639 & 74 & $\mathrm{~A}(-)$ & 2.225 & 1.355 \\
\hline 93 & $\mathrm{O}$ & 5.244 & 1.440 & 85 & $C(-)$ & 3.331 & 1.882 & 79 & $\mathrm{~A}(-)$ & 2.153 & 1.470 \\
\hline 52 & $\mathrm{E}$ & 5.238 & 1.344 & 97 & $\mathrm{E}(-)$ & 3.302 & 1.745 & 39 & $\mathrm{~A}(-)$ & 2.138 & 1.507 \\
\hline
\end{tabular}

Table 3

Summary Statistics for 120 IPIP-NEO Items

Note. $\mathrm{N}=516 ; \mathrm{I}=$ item number; $\mathrm{T}=$ trait measured; $\mathrm{M}=$ mean desirability; $\mathrm{SD}=$ standard deviation; $\mathrm{N}=$ Neuroticism; $\mathrm{E}=$ Extraversion; $\mathrm{O}=$ Openness to Experience; $\mathrm{A}=$ Agreeableness; $\mathrm{C}=$ Conscientiousness; $(-)=$ reversely keyed item. 
Correlations between $D$ and IIA estimates were sizable and ranged from - .948 to -.961 (Table 4). This result initially indicated that the item-pairs were consistently rank ordered between $D$ and IIA indices. Intercorrelations between IIA indices were also sizable and overall slightly higher, and ranged from .989 to .997.

\begin{tabular}{|c|c|c|c|c|c|c|c|c|}
\hline \multirow[b]{2}{*}{ Index } & \multicolumn{4}{|c|}{ Descriptives } & & \multicolumn{3}{|c|}{ Correlations } \\
\hline & M & $\mathrm{SD}$ & Min & Max & $\hat{D}$ & $B \hat{P}_{l}$ & $B \hat{P}_{q}$ & $A \hat{C}_{l}$ \\
\hline$\hat{D}$ & 1.534 & 1.102 & 0 & 4.242 & - & & & \\
\hline$B \hat{P}_{l}$ & 0.091 & 0.376 & -0.880 & 0.851 & -.958 & - & & \\
\hline$B \hat{P}_{q}$ & 0.036 & 0.571 & -1.680 & 0.929 & -.961 & .995 & - & \\
\hline$A \hat{C}_{l}$ & 0.158 & 0.379 & -0.706 & 0.901 & -.948 & .997 & .989 & - \\
\hline$A \hat{C}_{q}$ & 0.161 & 0.539 & -1.209 & 0.966 & -.953 & .993 & .996 & .994 \\
\hline
\end{tabular}

Table 4

Summary Statistics and Correlations for D and IIA Estimates Based on All IPIP-NEO Item-pairs Note. $\mathrm{N}=7,140 ; \mathrm{M}=$ mean; $\mathrm{SD}=$ standard deviation; $D=$ mean difference index; $B P_{l}=$ linearly weighted BP index (Brennan \& Prediger, 1981; Gwet, 2014); $B P_{q}=$ quadratically weighted BP index (Brennan \& Prediger, 1981; Gwet, 2014); $A C_{l}=$ linearly weighted $\mathrm{AC}_{2}$ index (Gwet, 2008; 2014); $A C_{q}=$ quadratically weighted $\mathrm{AC}_{2}$ index (Gwet, 2008; 2014).

To explore the relationship between $D$ and IIA indices further, we produced bivariate scatterplots of $D$ against each of the four IIA options. These plots are presented in Figure 1. We observe in the figure that the correspondence between $D$ and IIAs was high except at the lower end of the distribution of $D$ values, where the correspondence diverged markedly. This pattern of results was consistent across all IIA indices and strongly suggests that low $D$ values cannot be trusted to offer a consistent representation of actual similarity in desirability ratings. This result holds regardless of the choice of the cutoff value for $D$ in previous applications. Moreover, even if an extremely stringent $D$ cutoff value was used (e.g., $\hat{D}<0.01)$, the IIA for selected pairs 
would range from near perfect agreement for item-pair $\{\mathrm{i} 12, \mathrm{i} 20\}$ ("Take charge", "Work hard"), with $\hat{D}=0.006 ; B \hat{P}_{l}=0.816$ (95\% CI $\left.[0.789 ; 0.843]\right), B \hat{P}_{q}=0.916$ (95\% CI $\left.[0.892,0.939]\right)$, $A \hat{C}_{l}=0.873$ (95\% CI $\left.[0.853,0.893]\right)$, and $A \hat{C}_{q}=0.957$ (95\% CI $\left.[0.944,0.970]\right)$, to only slight agreement for item-pair $\{\mathrm{i} 76, \mathrm{i} 84\}$ ("Only feel comfortable with friends", "Have a high opinion of myself'), with $\hat{D}=0.008, B \hat{P}_{l}=0.127$ (95\% CI [0.065, 0.189]), $B \hat{P}_{q}=0.165$ (95\% CI [0.072, 0.259]), $A \hat{C}_{l}=0.180$ (95\% CI $\left.[0.114,0.245]\right)$, and $A \hat{C}_{q}==0.267$ (95\% CI $\left.[0.170,0.364]\right)$.
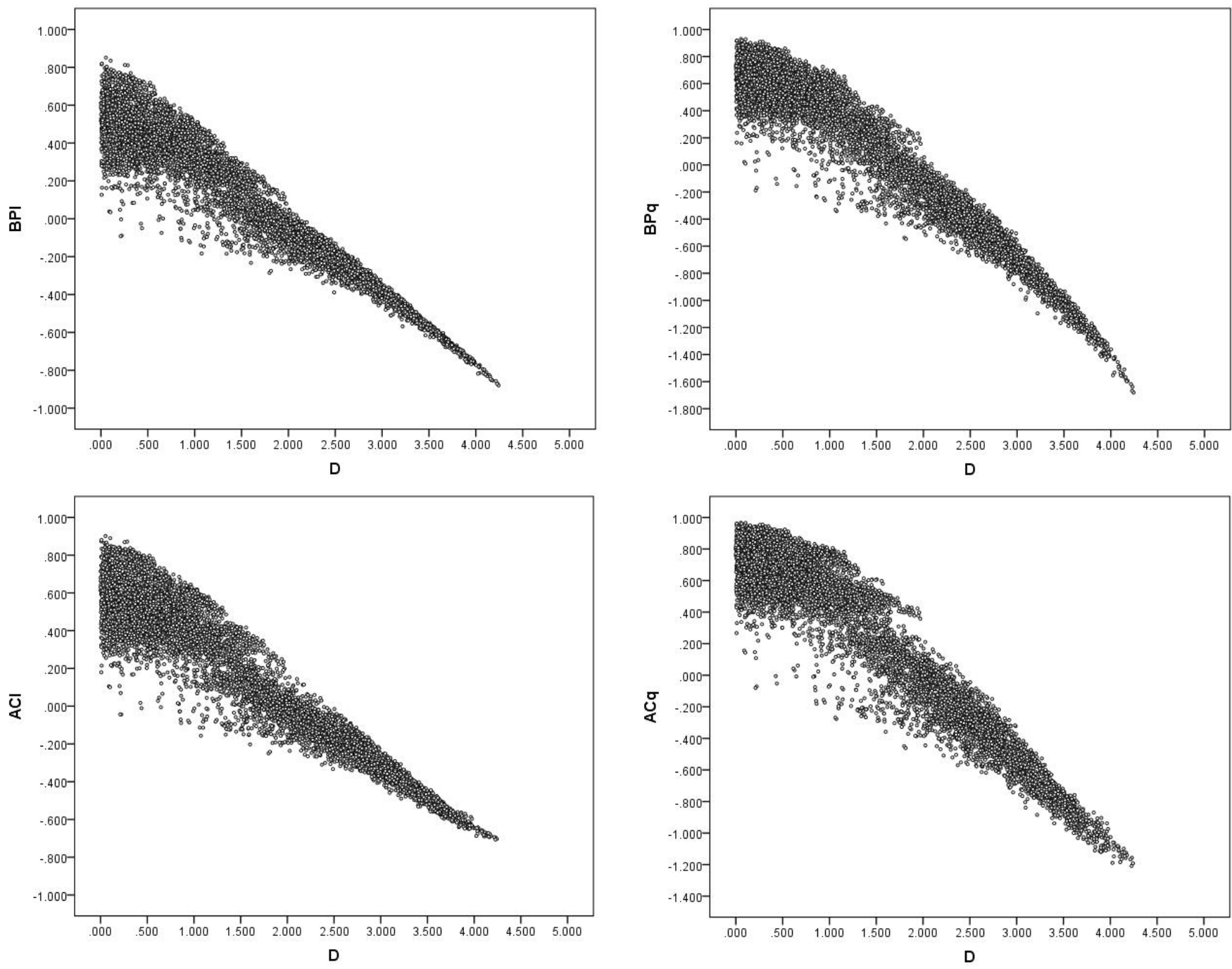

Figure 1. Bivariate scatterplots of the mean difference index $(D)$ against each inter-item agreement (IIA) index. 
Note. $B P_{l}=$ linearly weighted BP index (Brennan \& Prediger, 1981; Gwet, 2014); $B P_{q}=$ quadratically weighted BP index (Brennan \& Prediger, 1981; Gwet, 2014); $A C_{l}=$ linearly weighted $\mathrm{AC}_{2}$ index (Gwet, 2008; 2014); $A C_{q}$ = quadratically weighted $\mathrm{AC}_{2}$ index (Gwet, 2014).

Finally, we produced bivariate scatterplots for each combination of the four IIA indices to graphically depict correspondence among the measures. These plots are presented in Figure 2. Dotted lines in the plots represent reference lines of equal IIA values. In contrast to the relationships between IIAs and $D$ depicted in Figure 1, rank orderings of item-pairs appear relatively consistent between IIA indices across the entire range of IIA values in Figure 2. An effect of both index and weight on the resulting IIA values at high ends of IIA score distributions can also be observed in this figure. Specifically, there was a clear tendency for BP options and linear options to return somewhat smaller IIA estimates.

\section{Summary and Discussion}

Self-report measures of non-cognitive constructs in the forced-choice response format may prevent test takers from responding in a socially desirable way and result in improved assessment validity. However, if forced-choice item-blocks consist of indicator items that are different in terms of perceived desirability, negative consequences of SDR might not be reduced and potentially even enhanced. Accordingly, effective item matching is critical in SDR-resistant forced-choice test construction aimed at assessment across a broad range of situations and contexts. 

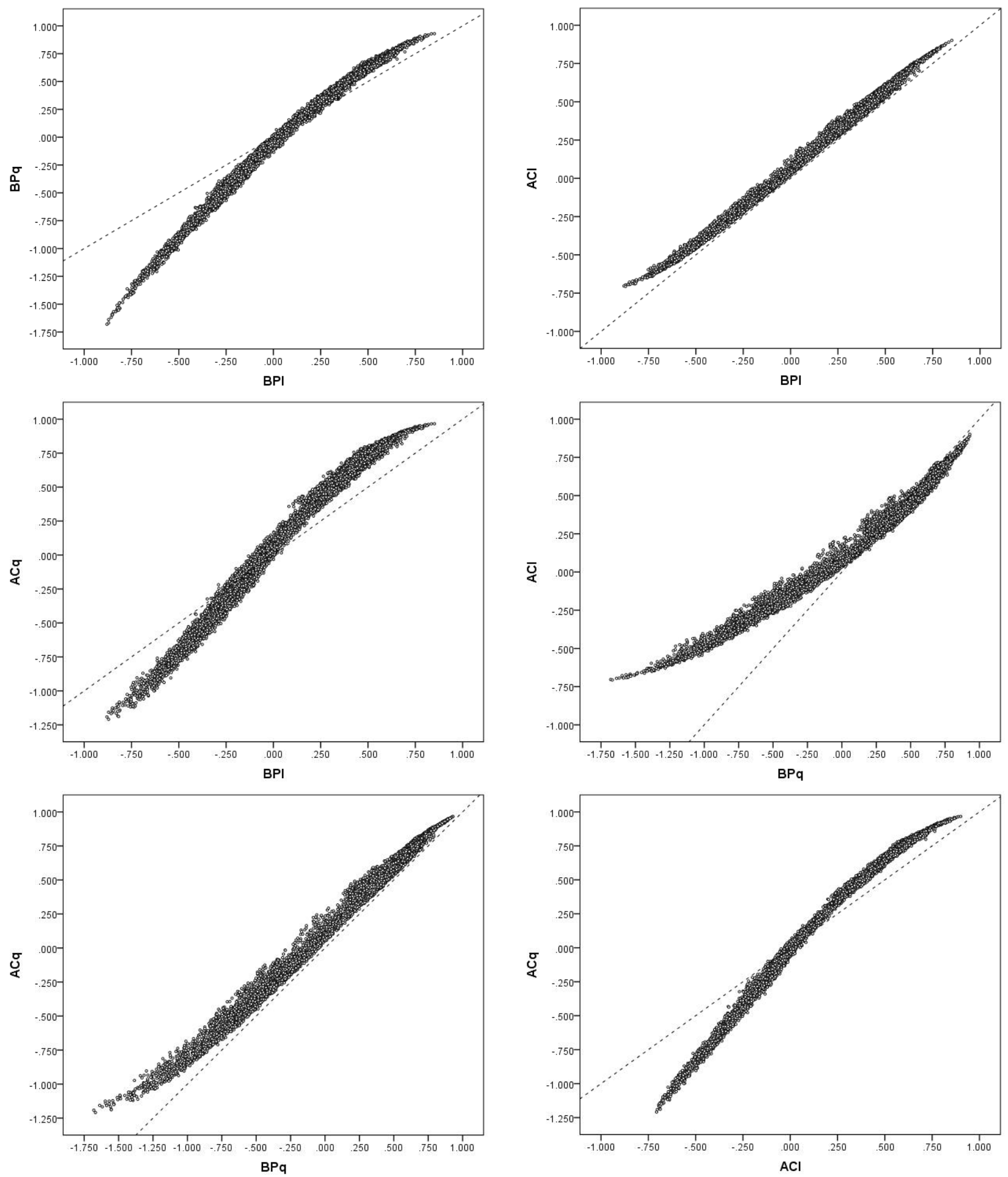

Figure 2. Bivariate scatterplots between each pair of inter-item agreement (IIA) indices.

Note. $B P_{l}=$ linearly weighted $\mathrm{BP}$ index (Brennan \& Prediger, 1981; Gwet, 2014); $B P_{q}=$ quadratically weighted BP index (Brennan \& Prediger, 1981; Gwet, 2014); $A C_{l}=$ linearly weighted $\mathrm{AC}_{2}$ index (Gwet, 2008; 2014); $A C_{q}$ = quadratically weighted $\mathrm{AC}_{2}$ index (Gwet, 2008; 2014). 
The standard procedure to match items in terms of desirability involves estimating the average desirability rating for each item under consideration. Absolute differences in item mean ratings $(D)$ are then used as an index of similarity between items in terms of desirability to drive the selection of "best" item combinations. Intuitively, smaller mean difference values indicate better item matches. The main disadvantage of the mean difference index is that it assumes variability of respondents' ratings around the mean can be attributed entirely to error. If this assumption is violated, $D$ may fail to appropriately quantify the similarity of desirability ratings between items and result, to extent, in mismatched items.

In this article, we have argued that an alternate focus on within-rater agreement with respect to desirability evaluations of each item-combination under consideration has the potential to circumvent problems in item desirability matching introduced by $D$. We reviewed several well-known absolute agreement indices potentially appropriate for item desirability matching and termed them inter-item agreement (IIA) indices in the context of our work. We proposed that the Brennan-Prediger index (Brennan \& Prediger, 1981; Gwet, 2014) and AC (Gwet, 2008, 2014) indices were superior alternatives to other mainstream IIA options, such as the intraclass correlation coefficient and weighted kappa, given peculiarities that yield misleading similarity values in certain circumstances with those measures.

We compared the traditional $D$ index and several IIA options on a set of empirically derived desirability ratings to evaluate practical concerns with the $D$ measure. We found an overall strong consistency between $D$ and IIA estimates, except at small $D$ values. This result is concerning, especially given that even when extremely stringent cutoff values of $D$ are used for item matching, the selection may involve item-pairs with a relatively poor IIA. These findings 
strongly indicate that relying on the mean difference index may fail to yield an optimal forcedchoice item-block assembly in terms of desirability. Accordingly, current findings reinforce conjectures that the lack of more convincing evidence in support of the forced-choice method as a SDR deterrent could be, at least partially, attributed to suboptimal item matching in previous research.

Although BP and AC indices can handle item-combinations involving any number of items, we have limited our discussion to a scenario involving only item-pairs. Traditionally, item desirability matching has relied on the mean difference between pairs of items because most of the forced-choice measures aimed at reducing SDR involved item-blocks consisting of item-pairs (e.g., Christiansen et al., 2005; Drasgow et al., 2012) or on tetrads composed of two equally desirable items and two equally undesirable items (e.g., Heggestad et al., 2006; Vasilopoulos et al., 2006). Our focus on pairwise similarity was thus natural to provide a direct comparison with the traditional mean difference index. Recently, a format consisting of three items (i.e., triads or triplets) per forced-choice block has been gaining in popularity (e.g., Guenole et al., 2018; Lee et al., 2019; Murano et al., 2021; Ng et al., 2021; Walton et al., 2020; Watrin et al., 2019; Wetzel \& Frick, 2020), because it seems to provide an optimal balance between the information gained and the cognitive burden placed on test takers. That said, future work should also consider similarity indices involving more than two items.

In the current article, we have discussed item matching procedures most frequently used in applications, i.e., those utilizing item desirability information retrieved in the rating format. The underlying assumption behind these procedures is that the perceived desirability of an item should not change when it is evaluated in the context of other items within a forced-choice block. If this assumption does not hold, item desirability matching might yield suboptimal item-block 
assembly regardless of the matching index discussed in this article. Although still limited, research evidence to date suggests that this concern might be warranted (see e.g., Lin \& Brown, 2017). If so, a conceptually more appropriate procedure would be to obtain comparative evaluations of the items' desirability (see e.g., Bürkner et al., 2019; Wetzel et al., 2021 for discussion), but its potential advantages over collecting desirability ratings are yet to be ascertained. The main challenge with this alternate approach, is that the number of itemcombinations for evaluation can be exceedingly large. Therefore, to keep participant burden at reasonable levels, selection of the most promising combinations must be carefully made $a$ priori, e.g., by utilizing item desirability ratings in concert with IIA indices proposed in this article.

In closing, while the main purpose of the current article was to discuss and advance the use of item desirability ratings, an equally important set of issues, related to methods to obtain these ratings, remains largely unanswered. Despite those issues being raised early in the forcedchoice method development (e.g., Waters, 1965), there has been both a lack of consistency and a lack of clarity in such guidelines in the consequent work. For example, 1) "What is the most adequate desirability sample composition?", 2) "What is the most effective type of instruction set to elicit item desirability evaluations?", and 3) "What are desirability sample size requirements?" are some of the outstanding questions, critical to item desirability matching, that lend themselves nicely to future empirical inquiry.

\subsection{Recommendations for applied researchers}

This study proposes alternatives to the mean difference index in rating-based item desirability matching. Although additional research is clearly needed in this area, several recommendations can be offered to applied researchers based on the current work. First, given strong assumptions underlying the mean difference index, we strongly encourage applied 
researchers to utilize IIA indices to quantify similarity in desirability between items instead. Among the IIA choices discussed, we cautiously recommend using the BP index in concert with linear weights $\left(\mathrm{BP}_{l}\right.$ in the article), as this option seems to yield somewhat more conservative similarity estimates. In terms of cutoff values for the minimum acceptable similarity level between items, we advise researchers to use the traditional 0.7 cutoff in the absence of better criteria. Finally, for those readers planning to adopt the IIA indices but also for those who remain interested in using the $D$ index to quantify similarity between items' desirability, we encourage them to incorporate uncertainty of the obtained estimates to justify their matching decisions further. Specifically, if IIA indices are used, items should be deemed of sufficient similarity only if the lower bound of the confidence interval of the respective IIA estimate falls above the chosen cut value. Conversely, if the $D$ index is used, the upper bound of the confidence interval should fall below the chosen cutoff. 


\section{References}

Anguiano-Carrasco, C., MacCann, C., Geiger, M., Seybert, J. M., \& Roberts, R. D. (2015).

Development of a forced-choice measure of typical-performance emotional intelligence.

Journal of Psychoeducational Assessment, 33(1), 83-97.

https://doi.org/10.1177/0734282914550387

Bartlett, C. J., \& Doorley, R. (1967). Social desirability response differences under research, simulated selection, and faking instructional sets. Personnel Psychology, 20(3), 281-288. https://doi.org/10.1111/j.1744-6570.1967.tb01524.x

Bass, B. M. (1957). Faking by sales applicants of a forced choice personality inventory. Journal of Applied Psychology, 41(6), 403-404. https://doi.org/10.1037/h0044670

Brennan, R. L., \& Prediger, D. J. (1981). Coefficient kappa: Some uses, misuses, and alternatives. Educational and Psychological Measurement, 41(3), 687-699. https://doi.org/10.1177/001316448104100307

Brown, R. D., \& Hauenstein, N. M. (2005). Interrater agreement reconsidered: An alternative to the rwg indices. Organizational Research Methods, 8(2), 165-184. https://doi.org/10.1177/1094428105275376

Brown, A., \& Maydeu-Olivares, A. (2011). Item response modeling of forced-choice questionnaires. Educational and Psychological Measurement, 71(3), 460-502. http://dx.doi.org/10.1177/0013164410375112

Burke, M. J., Finkelstein, L. M., \& Dusig, M. S. (1999). On average deviation indices for estimating interrater agreement. Organizational Research Methods, 2(1), 49-68. https://doi.org/10.1177/109442819921004 
Bürkner, P. C., Schulte, N., \& Holling, H. (2019). On the statistical and practical limitations of Thurstonian IRT models. Educational and Psychological Measurement, 79(5), 827-854. https://doi.org/10.1177/0013164419832063

Chernyshenko, O. S., Stark, S., Prewett, M. S., Gray, A. A., Stilson, F. R., \& Tuttle, M. D. (2009). Normative scoring of multidimensional pairwise preference personality scales using IRT: Empirical comparisons with other formats. Human Performance, 22(2), $105-$ 127. https://doi.org/10.1080/08959280902743303

Cheung, M. W. L., \& Chan, W. (2002). Reducing uniform response bias with ipsative measurement in multiple-group confirmatory factor analysis. Structural Equation Modeling, 9(1), 55-77. https://doi.org/10.1207/S15328007SEM0901_4

Christiansen, N. D., Burns, G. N., \& Montgomery, G. E. (2005). Reconsidering forced-choice item formats for applicant personality assessment. Human Performance, 18(3), 267-307. http://dx.doi.org/10.1207/s15327043hup1803_4

Cohen, J. (1968). Weighted kappa: Nominal scale agreement provision for scaled disagreement or partial credit. Psychological Bulletin, 70(4), 213-220. https://doi.org/10.1037/h0026256

Converse, P. D., Pathak, J., Quist, J., Merbedone, M., Gotlib, T., \& Kostic, E. (2010). Statement desirability ratings in forced-choice personality measure development: Implications for reducing score inflation and providing trait-level information. Human Performance, 23(4), 323-342. https://doi.org/10.1080/08959285.2010.501047

Crowne, D. P., \& Marlowe, D. (1960). A new scale of social desirability independent of psychopathology. Journal of Consulting Psychology, 24(4), 349-354. https://doi.org/10.1037/h0047358 
Dilchert, S., \& Ones, D. S. (2011). Application of preventive strategies. In M. Ziegler, C. MacCann \& D.R. Roberts (Eds.), New perspectives on faking in personality assessment (pp. 177-200). New York: Oxford University Press.

Drasgow, F., Stark, S., Chernyshenko, O. S., Nye, C. D., Hulin, C. L., \& White, L. A. (2012). Development of the tailored adaptive personality assessment system (TAPAS) to support army personnel selection and classification decisions. (Technical Report No. 1311). Arlington, VA: U.S. Army Research Institute for the Behavioral and Social Sciences. Retrieved from https://apps.dtic.mil/docs/citations/ADA564422

Dueber, D. M., Love, A. M., Toland, M. D., \& Turner, T. A. (2019). Comparison of singleresponse format and forced-choice format instruments using Thurstonian item response theory. Educational and Psychological Measurement, 79(1), 108-128. https://doi.org/10.1177\%2F0013164417752782

Edwards, A. L. (1953). The relationship between the judged desirability of a trait and the probability that the trait will be endorsed. Journal of Applied Psychology, 37(2), 90-93. https://doi.org/10.1037/h0058073

Edwards, A. L. (1957). The social desirability variable in personality assessment and research. New York: Dryden.

Feingold, A. (1994). Gender differences in personality: A meta-analysis. Psychological Bulletin, 116(3), 429-456. https://doi.org/10.1037/0033-2909.116.3.429

Feinstein, A. R., \& Cicchetti, D. V. (1990). High agreement but low kappa: I. The problems of two paradoxes. Journal of Clinical Epidemiology, 43(6), 543-549. https://doi.org/10.1016/0895-4356(90)90158-L 
Feldman, M. J., \& Corah, N. L. (1960). Social desirability and the forced choice method. Journal of Consulting Psychology, 24(6), 480-482. https://doi.org/10.1037/h0042687

Fleiss, J. L. (1981). Statistical methods for rates and proportions. New York: Wiley \& Sons.

Fleiss, J. L., \& Cohen, J. (1973). The equivalence of weighted kappa and the intraclass correlation coefficient as measures of reliability. Educational and Psychological Measurement, 33(3), 613-619. https://doi.org/10.1177/001316447303300309

Goldberg, L. R. (1990). An alternative "description of personality": The big-five factor structure. Journal of Personality and Social Psychology, 59(6), 1216-1229. https://doi.org/10.1037/0022-3514.59.6.1216

Goldberg, L. R. (1992). The development of markers for the Big-Five factor structure. Psychological Assessment, 4(1), 26-42. https://doi.org/10.1037/1040-3590.4.1.26

Graham, P., \& Jackson, R. (1993). The analysis of ordinal agreement data: Beyond weighted kappa. Journal of Clinical Epidemiology, 46(9), 1055-1062. https://doi.org/10.1016/0895-4356(93)90173-X

Guenole, N., Brown, A. A., \& Cooper, A. J. (2018). Forced-choice assessment of work-related maladaptive personality traits: Preliminary evidence from an application of Thurstonian item response modeling. Assessment, 25(4), 513-526. https://doi.org/10.1177/1073191116641181

Gwet, K. L. (2008). Computing inter- rater reliability and its variance in the presence of high agreement. British Journal of Mathematical and Statistical Psychology, 61(1), 29-48. https://doi.org/10.1348/000711006X126600 
Gwet, K. L. (2014). Handbook of inter-rater reliability ( $4^{\text {th }}$ ed.): The definitive guide to measuring the extent of agreement among raters. Gaithersburg, MD: Advanced Analytics Press.

Heggestad, E. D., Morrison, M., Reeve, C. L., \& McCloy, R. A. (2006). Forced-choice assessments of personality for selection: Evaluating issues of normative assessment and faking resistance. Journal of Applied Psychology, 91(1), 9-24. https://doi.org/10.1037/0021-9010.91.1.9

Hedberg, R., \& Baxter, B. (1963). Favorableness ratings of forced-choice statements: Applicants vs. non-applicants. Personnel Psychology, 16(1), 23-27. https://doi.org/10.1111/j.17446570.1963.tb01251.x

Jackson, D. N., Wroblewski, V. R., \& Ashton, M. C. (2000). The impact of faking on employment tests: Does forced choice offer a solution?. Human Performance, 13(4), 371388. https://doi.org/10.1207/S15327043HUP1304_3

James, L. R., Demaree, R. G., \& Wolf, G. (1984). Estimating within-group interrater reliability with and without response bias. Journal of Applied Psychology, 69(1), 85-98. https://doi.org/10.1037/0021-9010.69.1.85

Johnson, J. A. (2014). Measuring thirty facets of the Five Factor Model with a 120-item public domain inventory: Development of the IPIP-NEO-120. Journal of Research in Personality, 51, 78-89. https://doi.org/10.1016/j.jrp.2014.05.003

Kilmann, R. H., \& Thomas, K. W. (1977). Developing a forced-choice measure of conflicthandling behavior: The" MODE" instrument. Educational and Psychological Measurement, 37(2), 309-325. https://doi.org/10.1177/001316447703700204 
Klein, D. (2018). Implementing a general framework for assessing interrater agreement in Stata. The Stata Journal, 18(4), 871-901. https://doi.org/10.1177/1536867X1801800408

Landis, J. R., \& Koch, G. G. (1977). The measurement of observer agreement for categorical data. Biometrics, 33(1), 159-174. https://doi.org/10.2307/2529310

LeBreton, J. M., Burgess, J. R., Kaiser, R. B., Atchley, E. K., \& James, L. R. (2003). The restriction of variance hypothesis and interrater reliability and agreement: Are ratings from multiple sources really dissimilar?. Organizational Research Methods, 6(1), 80-128. https://doi.org/10.1177/1094428102239427

LeBreton, J. M., \& Senter, J. L. (2008). Answers to 20 questions about interrater reliability and interrater agreement. Organizational Research Methods, 11(4), 815-852. https://doi.org/10.1177/1094428106296642

Lee, P., Joo, S.-H., Stark, S., \& Chernyshenko, O. S. (2019). GGUM-RANK statement and person parameter estimation with multidimensional forced choice triplets. Applied Psychological Measurement, 43(3), 226-240. https://doi.org/10.1177/0146621618768294

Lin, Y., \& Brown, A. (2017). Influence of context on item parameters in forced-choice personality assessments. Educational and Psychological Measurement, 77(3), 389-414. https://doi.org/10.1177/0013164416646162

Longstaff, H. P., \& Jurgensen, C. E. (1953). Fakability of the Jurgensen Classification Inventory. Journal of Applied Psychology, 37(2), 86-89. https://doi.org/10.1037/h0057806

Maher, H. (1959). Studies of transparency in forced-choice scales: I. Evidence of transparency. Journal of Applied Psychology, 43(4), 275-278. https://doi.org/10.1037/h0046315

Meade, A. W., \& Craig, S. B. (2012). Identifying careless responses in survey data. Psychological Methods, 17(3), 437-455. https://doi.org/10.1037/a0028085 
Murano, D., Lipnevich, A. A., Walton, K. E., Burrus, J., Way, J. D., \& Anguiano-Carrasco, C. (2021). Measuring social and emotional skills: Development of self-report big five Likert, situational judgment test, and forced choice items for elementary school students. Personality and Individual Differences. Advance online publication. https://doi.org/10.1016/j.paid.2020.110012

Naemi, B., Seybert, J., Robbins, S., \& Kyllonen, P. (2014). Examining the WorkFORCETM assessment for job fit and core capabilities of FACETS ${ }^{\text {TM }}$. ETS Research Report Series, 2014(2), 1-43. https://doi.org/10.1002/ets2.12040

Nederhof, A. J. (1985). Methods of coping with social desirability bias: A review. European Journal of Social Psychology, 15(3), 263-280. https://doi.org/10.1002/ejsp.2420150303

Ng, V., Lee, P., Ho, M. H. R., Kuykendall, L., Stark, S., \& Tay, L. (2021). The development and validation of a multidimensional forced-choice format character measure: Testing the Thurstonian IRT approach. Journal of Personality Assessment, 103(2), 224-237. https://doi.org/10.1080/00223891.2020.1739056

Norman, W. T. (1963). Personality measurement, faking, and detection: An assessment method for use in personnel selection. Journal of Applied Psychology, 47(4), 225-241. https://doi.org/10.1037/h0042106.

Paulhus, D. L. (1984). Two-component models of socially desirable responding. Journal of Personality and Social Psychology, 46(3), 598-609. https://doi.org/10.1037/00223514.46.3.598

Paulhus, D. L., \& Vazire, S. (2007). The self-report method. In R. W. Robins, R. C. Fraley, \& R. F. Krueger (Eds.), Handbook of research methods in personality psychology (pp. 224239). New York, NY: Guilford Press. 
Quarfoot, D., \& Levine, R. A. (2016). How robust are multirater interrater reliability indices to changes in frequency distribution?. The American Statistician, 70(4), 373-384. https://doi.org/10.1080/00031305.2016.1141708

R Core Team. (2018). R: A language and environment for statistical computing. R Foundation for Statistical Computing, Vienna, Austria. https://www.r-project.org/

Rothstein, M. G., \& Goffin, R. D. (2006). The use of personality measures in personnel selection: What does current research support?. Human Resource Management Review, 16(2), 155-180. https://doi.org/10.1016/j.hrmr.2006.03.004

Saltz, E., Reece, M., \& Ager, J. (1962). Studies of forced-choice methodology: Individual differences in social desirability. Educational and Psychological Measurement, 22(2), 365-370. https://doi.org/10.1177/2F001316446202200209

Schuster, C. (2004). A note on the interpretation of weighted kappa and its relations to other rater agreement statistics for metric scales. Educational and Psychological Measurement, 64(2), 243-253. https://doi.org/10.1177/0013164403260197

Shrout, P. E. (1998). Measurement reliability and agreement in psychiatry. Statistical Methods in Medical Research, 7(3), 301-317. https://doi.org/10.1177/096228029800700306

Shrout, P. E., \& Fleiss, J. L. (1979). Intraclass correlations: Uses in assessing rater reliability. Psychological Bulletin, 86(2), 420-428. https://doi.org/10.1037/0033-2909.86.2.420

Stark, S., Chernyshenko, O. S., \& Drasgow, F. (2005). An IRT approach to constructing and scoring pairwise preference items involving stimuli on different dimensions: The multiunidimensional pairwise-preference model. Applied Psychological Measurement, 29(3), 184-203. http://dx.doi.org/10.1177/0146621604273988 
Stark, S., Chernyshenko, O. S., Drasgow, F., Nye, C. D., White, L. A., Heffner, T., \& Farmer, W. L. (2014). From ABLE to TAPAS: A new generation of personality tests to support military selection and classification decisions. Military Psychology, 26(3), 153-164. https://doi.org/10.1037/mil0000044

Tran, D., Dolgun, A., \& Demirhan, H. (2020). Weighted inter-rater agreement measures for ordinal outcomes. Communications in Statistics-Simulation and Computation, 49(4), 9891003. https://doi.org/10.1080/03610918.2018.1490428

Usami, S., Sakamoto, A., Naito, J., \& Abe, Y. (2016). Developing pairwise preference-based personality test and experimental investigation of its resistance to faking effect by item response model. International Journal of Testing, 16(4), 288-309. http://dx.doi.org/10.1080/15305058.2016.1145123

Vasilopoulos, N. L., Cucina, J. M., Dyomina, N. V., Morewitz, C. L., \& Reilly, R. R. (2006). Forced-Choice personality tests: A measure of personality and cognitive ability? Human Performance, 19(3), 175-199. http://dx.doi.org/10.1207/s15327043hup1903_1

Walton, K. E., Cherkasova, L., \& Roberts, R. D. (2020). On the validity of forced choice scores derived from the Thurstonian item response theory model. Assessment, 27(4), 706-718. https://doi.org/10.1177/1073191119843585

Warrens, M. J. (2012). Some paradoxical results for the quadratically weighted kappa. Psychometrika, 77(2), 315-323. https://doi.org/10.1007/s11336-012-9258-4

Waters, L. K. (1965). A note on the "fakability" of forced-choice scales. Personnel Psychology, 18(2), 187-191. https://doi.org/10.1111/j.1744-6570.1965.tb00277.x 
Watrin, L., Geiger, M., Spengler, M., \& Wilhelm, O. (2019). Forced-choice versus Likert responses on an occupational Big Five questionnaire. Journal of Individual Differences, 40(3), 134-148. https://doi.org/10.1027/1614-0001/a000285

Wetzel, E., Böhnke, J. R., \& Brown, A. (2016). Response biases. In F. T. L. Leong, D. Bartram, F. Cheung, K. F. Geisinger, \& D. Iliescu (Eds.). The ITC international handbook of testing and assessment (pp. 349-363). Oxford University Press, New York.

Wetzel, E., \& Frick, S. (2020). Comparing the validity of trait estimates from the multidimensional forced-choice format and the rating scale format. Psychological Assessment, 32(3), 239-253. https://doi.org/10.1037/pas0000781

Wetzel, E., Frick, S., Brown, A. (2021). Does multidimensional forced-choice prevent faking? Comparing the susceptibility of the multidimensional forced-choice format and the rating scale format to faking. Psychological Assessment. 33(2), 156-170. https://doi.org/10.1037/pas0000971

Wongpakaran, N., Wongpakaran, T., Wedding, D., \& Gwet, K. L. (2013). A comparison of Cohen's Kappa and Gwet's AC1 when calculating inter-rater reliability coefficients: a study conducted with personality disorder samples. BMC Medical Research Methodology, 13(1), 61. https://doi.org/10.1186/1471-2288-13-61

\section{Funding}

This research did not receive any specific grant from funding agencies in the public, commercial, or not-for-profit sectors. 